\title{
Application of Multilayer Planar Waveguide Structures to Sensing
}

\author{
E. AuguściuK* And D. DZiąG
}

Faculty of Physics, Warsaw University of Technology, Koszykowa 75, 00-662 Warsaw, Poland

The multilayer planar step index waveguides have been studied in detail for many years now. We examined gradient index waveguide, which was not thoroughly studied. In this article we have studied structures made from three, four, and five layers. We also used different substrates for this experiment. Gradient index waveguides were made in Bk7 and Gevert's glass by the ion-exchange method. Then we put on it a thin layer of polymer and examined it again. Afterwards we applied a second layer of polymer achieving five-layer planar waveguide. Layers deposited on gradient index waveguide change the propagating conditions of light beam in waveguide structures. Using a generalized $m$-line spectroscopy method we determine thickness and refractive index of each layer of waveguide structure. In the next step, a simulation for step index planar waveguides was run. The values for each layer were taken from previously calculated thickness and refractive index for multilayer gradient index waveguides. Beam propagation method was used to obtain $N_{\text {eff }}$ only for step index waveguide structures to compare with $N_{\text {eff }}$ of gradient index waveguide structure. The changes in propagation of a light beam not only in waveguide (several modes) layer may be applied to sensing and controlling the direction of light in the waveguide structure (by depositing on it a polymer layer with the appropriate refractive index).

PACS numbers: 42.79.Gn, 42.82.Ds

\section{Introduction}

In the paper a determination of optical properties of multilayer structures has been performed. We examined gradient index waveguides made by the ion-exchange method. In the process the time of exchange has influenced the thickness of the waveguide. Number of propagating modes in a gradient waveguide was changing due to the thickness of used waveguides. Second parameter (which we control) was a temperature, which changes refractive index of the waveguide.

Using $m$-line spectroscopy method only parameters (refractive index and thickness) of waveguide (three-layer structure) may be evaluated [1, 2]. However, generalized $m$-line method allows evaluating parameters of thin layers deposited on the waveguide [3, 4].

The deposition of layers on the waveguide changes propagating conditions in the structure. After obtaining thickness and refractive index of investigated structures we run a simulation using calculated values. Beam propagation method (BPM) method gave us a mode structure only for step index waveguide and the waveguide with one and two polymer layers.

Our goal was the determination of influence of different polymer layers on propagation in the gradient waveguide structure.

\section{Theoretical background}

\subsection{Determination of parameters} of gradient index waveguide

Figure 1 presents a gradient index waveguide and its refractive index profile.

\footnotetext{
* corresponding author; e-mail: eaugust@if.pw.edu.pl
}

Because of the refractive index profile of the waveguide (Fig. 1) we used a dispersion equation written as [2]:

$$
\int_{0}^{x_{m}} \sqrt{n^{2}(0)-N_{\text {eff }}^{2}} \mathrm{~d} x=F_{m},
$$

where

$$
\begin{aligned}
F_{m} & =\frac{1}{k_{0}}\left[\arctan \left(\left(\frac{n^{2}(0)}{n_{2}^{2}}\right)^{\rho} \sqrt{\frac{\alpha_{m}+n_{0}^{2}-n_{2}^{2}}{n^{2}(0)-\alpha_{m}-n_{0}^{2}}}\right)\right. \\
+ & \left.\frac{\pi}{4}+m \pi\right],
\end{aligned}
$$

$m$ - number of mode, $X_{m}-m$-th return point, $k_{0}$ wave number, $\lambda$ - wavelength, $n(x)$ - refractive index profile of the waveguide, $n(0)$ - refractive index of the waveguide on upper surface, $n_{2}$ - medium index of refraction, $n_{0}$ - substrate index of refraction,

$$
\begin{aligned}
& \alpha_{m}=N_{\text {eff }}^{2}-n_{0}^{2}, \\
& \rho=\left\{\begin{array}{lll}
0 & \text { for } & \text { TE }, \\
1 & \text { for } & \text { TM. }
\end{array}\right.
\end{aligned}
$$

To obtain thickness we used an equation

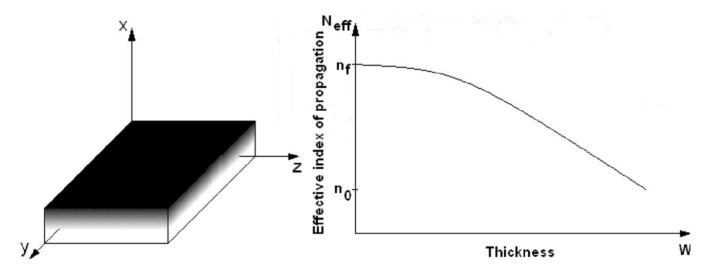

Fig. 1. The gradient index waveguide and the refractive index profile. 


$$
\begin{aligned}
& x(f)=\frac{2}{\pi} \int_{f(0)}^{f(x)}\left(\frac{\frac{\mathrm{d} F(\alpha)}{\mathrm{d} \alpha_{m}}}{\sqrt{\alpha-f(x)}}\right) \mathrm{d} \alpha, \\
& f(x)=n^{2}(x)-n_{0}^{2} .
\end{aligned}
$$

$F(\alpha)$ is a polynomial continuous function,

$$
n(0)=N_{-0.75} \text {, }
$$

which means that the refractive index on the waveguide surface is determined by effective index for mode $m=$ -0.75 .

\subsection{Determination of parameters of thin layers deposited on the waveguide}

Figure 2 presents the waveguide with deposited thin polymer layer on it (four-layer structure).

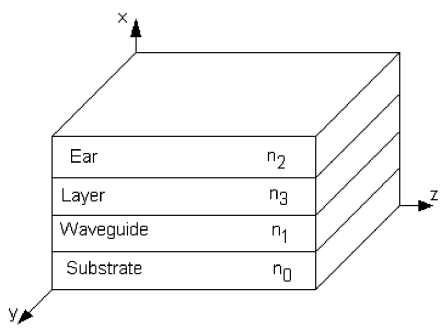

Fig. 2. The four-layer structure.

To determine the thickness and refractive index of the gradient index waveguide with one layer (Fig. 2) [5] we need to analyze two possibilities for this case. First, $n_{3}<$ $N_{\text {eff }}$, the polymer layer has a lower refractive index than the refractive index of propagation. Second, $n_{3}>N_{\text {eff }}$, the polymer layer has higher refractive index than the refractive index of propagation.

In the first case dispersion equation can be written as [3]:

$$
\begin{aligned}
& b X_{m}=\arctan \left(\frac{p_{s}}{b}\right) \\
& \quad+\arctan \left(\frac{p_{l}}{b} \frac{p_{\text {med }} \cosh \left(p_{l} t\right)+p_{l} \sinh \left(p_{l} t\right)}{p_{l} \cosh \left(p_{l} t\right)+p_{\text {med }} \sinh \left(p_{l} t\right)}\right)+m \pi,
\end{aligned}
$$

where

$$
\begin{aligned}
& p_{l}=\sqrt{N_{\mathrm{eff}}^{\prime \prime 2}-n_{3}^{2}}, \quad p_{\mathrm{med}}=\sqrt{N_{\mathrm{eff}}^{\prime \prime 2}-n_{2}^{2}}, \\
& p_{s}=\sqrt{N_{\mathrm{eff}}^{\prime \prime 2}-n_{0}^{2}},
\end{aligned}
$$

$n_{3}$ - refractive index of thin layer, $t$ - thickness of thin layer, $N_{\text {eff }}^{\prime \prime}$ - effective index of propagation for four-layer structure.

Left side of Eq. (8) for the structure with the gradient index waveguide can be written as [5]:

$$
b X_{m}=k_{0} \int_{0}^{X_{m}} \sqrt{n^{2}(x)-N_{\text {eff }}^{\prime \prime 2}} \mathrm{~d} x
$$

and

$$
b=\frac{k_{0} \int_{0}^{X_{m}} \sqrt{n^{2}(x)-N_{\mathrm{eff}}^{\prime \prime 2}} \mathrm{~d} x}{X_{m}} .
$$

We can calculate $X_{m}$ from the inverse function of profile

$$
\begin{aligned}
& n^{-1}(x)=x(n), \\
& X_{m}=x\left(N_{\text {eff }}^{\prime \prime}\right) .
\end{aligned}
$$

Transformation of Eq. (8) gave

$$
t=\frac{1}{p_{l}}\left[\operatorname{arccoth}\left(\frac{p_{l}^{2}-p_{\text {med }} b \tan C}{p_{l}\left(b \tan C-p_{\text {med }}\right)}\right)\right],
$$

where

$$
C=b X_{m}-m \pi-\arctan \left(\frac{p_{s}}{b_{1}}\right) .
$$

In the second case $\left(n_{3}>N_{\text {eff }}\right)$ dispersion equation looks like

$$
\begin{aligned}
& b X_{m}=\arctan \left(\frac{p_{s}^{\prime \prime}}{b}\right) \\
& +\arctan \left(\frac{p_{l}}{b} \frac{p_{\text {med }} \cos \left(p_{l}^{\prime \prime} t\right)+p_{l}^{\prime \prime} \sin \left(p_{l}^{\prime \prime} t\right)}{p_{l}^{\prime \prime} \cos \left(p_{l}^{\prime \prime} t\right)+p_{\text {med }} \sin \left(p_{l}^{\prime \prime} t\right)}\right)+m \pi,
\end{aligned}
$$

where $p_{l}^{\prime \prime}=\sqrt{n_{3}^{2}-N_{\text {eff }}^{2}}$ becomes imaginary.

Thickness of thin layer can be obtained from

$$
t=\frac{1}{p_{l}}\left[\operatorname{arccot}\left(\frac{p_{l}^{\prime \prime 2}-p_{\text {med }} b \tan C}{p_{l}^{\prime \prime}\left(b \tan C-p_{\text {med }}\right)}\right)+j \pi\right],
$$

where

$$
C=b X_{m}-m \pi-\arctan \left(\frac{p_{s}}{b}\right), \quad j \in Z .
$$

\subsection{Multilayer waveguide structure}

Figure 3 presents the waveguide with two thin layers deposited on it (five-layer structure).

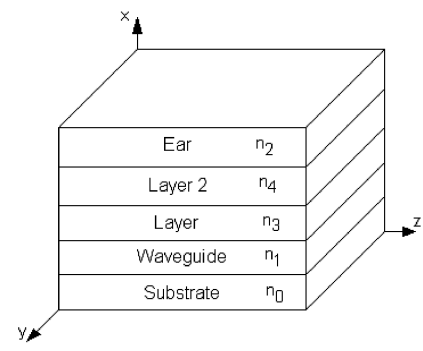

Fig. 3. The five-layer structure.

In this structure electrical field of any waveguiding layer possesses a component

$$
\begin{gathered}
E_{y j}(x, z)=A_{j} \exp \left(\mathrm{i}\left(\gamma_{j} x+\beta_{m} z\right)\right) \\
+B_{j} \exp \left(\mathrm{i}\left(-\gamma_{j} x+\beta_{m} z\right)\right)
\end{gathered}
$$

and the tangential component of magnetic field can be written as

$$
H_{z j}(x, z)=\mathrm{i}\left(\omega \mu_{0}\right)^{-1} \frac{\partial E_{y j}}{\partial x}
$$

where $j$ - for suitable layer $(0,1,2,3,4), \omega$ - angular 
frequency, $\mu_{0}$ - magnetic inductive capacity in vacuum, $\beta_{m}=k N_{\text {eff }}$ - propagation constant for $m$-th mode.

The values as thickness and refractive index for a five-layer structure (Fig. 3) [6] can be calculated from transfer matrix $M_{j}$, which binds the electromagnetic fields at the backplane of the layer to the fields at its frontplane, and can be associated with each layer

$$
M_{j}=\left(\begin{array}{cc}
\cos \left(\omega \mu_{0} \gamma_{j} d_{j}\right) & \frac{\mathrm{i}}{\gamma_{j}} \sin \left(\omega \mu_{0} \gamma_{j} d_{j}\right) \\
\mathrm{i} \gamma_{j} \sin \left(\omega \mu_{0} \gamma_{j} d_{j}\right) & \cos \left(\omega \mu_{0} \gamma_{j} d_{j}\right)
\end{array}\right) \text {, }
$$

where $d_{j}$ - thickness of $j$ layer, $\gamma_{j}=k\left(\omega \mu_{0}\right)^{-1} \mid n_{j}^{2}-$ $\left.N_{\text {eff }}^{2}\right|^{0.5}$ is for propagating wave in layer $j, \gamma_{j}=$ $\mathrm{i} k\left(\omega \mu_{0}\right)^{-1}\left|n_{j}^{2}-N_{\text {eff }}^{2}\right|^{0.5}$ is for evanescent wave in layer $j$.

In the interface of the layer the tangential component of the magnetic and electric fields must be continuous at the interface of the layers. These conditions together with the condition for obtaining guiding lead to

$$
\left(\begin{array}{c}
1 \\
\gamma_{2}
\end{array}\right) E_{2 y}=M_{4} M_{3} M_{1}\left(\begin{array}{c}
1 \\
\gamma_{0}
\end{array}\right) E_{0 y},
$$

which has solutions only for

$$
\gamma_{2} m_{11}+\gamma_{2} \gamma_{0} m_{12}+m_{21}+\gamma_{0} m_{22}=0,
$$

where $m_{i j}$ are the components of the matrix $M$.

\section{Experimental arrangement}

\subsection{Preparation of structures}

For measurements the planar gradient index waveguides were used. As a substrate we used two kinds of glass: $\operatorname{Bk} 7\left(n_{0}=1.51510 \pm 0.00002\right)$ and Gevert's $\left(n_{0}=1.50450 \pm 0.00002\right)$. The gradient index waveguides (named three-layer structure) have been produced by ion-exchange method in the glass substrate (Fig. 1). In the process, the exchange occurs between cations of $\mathrm{Ag}$ and $\mathrm{K}$ (from substances $\mathrm{KNO}_{3}$ and $\mathrm{AgNO}_{3}$ ) and cations $\mathrm{Na}$ from the glass. The thickness of the waveguide was determined in dependence on the time of the ion-exchange.

In the next step we deposited on the waveguide (by spin-coating method) the first layer of two kinds of polymers: polystyrene (PS) and polyvinyl acetate (PVAC). This way we created four-layer structures (Fig. 2).

The second layer was put on the first layer by spin-coating method (five-layer structure shown in Fig. 3). Polymers used in this stage are polyvinylidene fluoride (PVDF) and polyimide.

We put polymers on layers in three combinations (layer 1, then layer 2): $\mathrm{PS}+\mathrm{PVDF}, \mathrm{PVAC}+$ polyimide and PS+polyimide. The values of refractive index of used polymers are shown in Table I [7, 8].

\subsection{Measurement by m-line spectroscopy method}

The setup of $m$-line spectroscopy has been used to measure coupling angles of laser beam to the waveguide as shown in Fig. 4.

Laser light (He-Ne with wavelength $\lambda=632.8 \mathrm{~nm}$ ) is coupled into the waveguide by the prism. The using of a

\section{TABLE I}

Refractive index of bulk polymers used as a layer.

\begin{tabular}{c|c}
\hline \hline & Refractive index \\
\hline PVDF & $1.420 \pm 0.001$ \\
polyimide & $1.700 \pm 0.001$ \\
PS & $1.589 \pm 0.001$ \\
PVAC & $1.466 \pm 0.001$
\end{tabular}

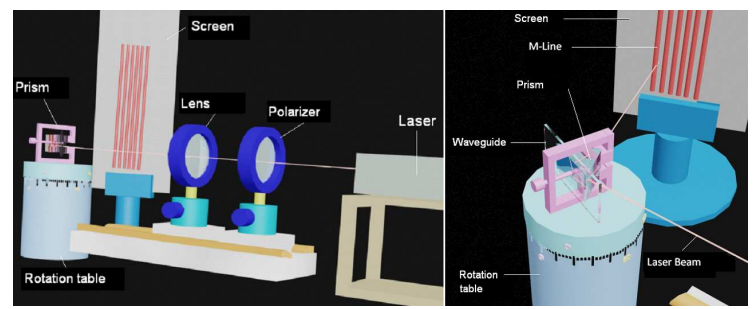

Fig. 4. Setup for $m$-line spectroscopy.

symmetrical prism causes a reverse effect, too, that is the decoupling of the light beam, but now in the form of bright lines ( $m$-modes) observed on the screen. By measuring the coupling angles we can determine - out of a simple geometric dependence [4] — effective propagation indices in the waveguide.

The changes of the coupling angle after deposition of the thin layer are by one order larger as compared with measurement accuracy of $0.5^{\prime}$ - effective propagation index in such a waveguide structure is different and is changing dependently on the refractive index of deposited thin layer.

\section{Results from experiment}

For every measured waveguide structure $[8,9]$ the thickness of all layers was calculated as well as their refractive index. Incident angles are used to calculate effective indices of propagation. First we analyzed effective index of propagation of gradient index waveguide, then with first layer and with both layers. Then we simulated, using BPM method, a step index multilayer planar waveguide. Thicknesses and refractive indices used in BPM method were taken from calculated values of gradient index waveguide structures as an assumption. We compared multilayer gradient index waveguide structures with multilayer step index waveguide structures.

The article presents several examples of the examined waveguide structures.

As the first structure to analyze we choose the waveguide with combination PS and PVDF polymer layers. Gevert's glass is used as a substrate. Evaluated data from this structure are shown in Fig. 5. Theoretical values for each layer of step index waveguide structure are shown in Fig. 6. Table II contains the calculated thickness and refractive index of each layer. 


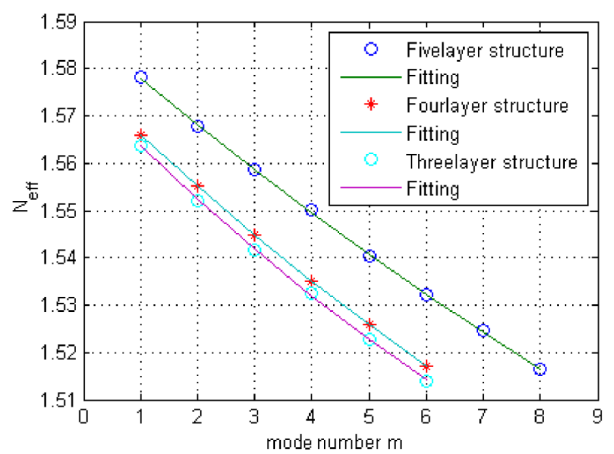

Fig. 5. Experimental data for structure no. 1 made in Gevert's glass substrate.

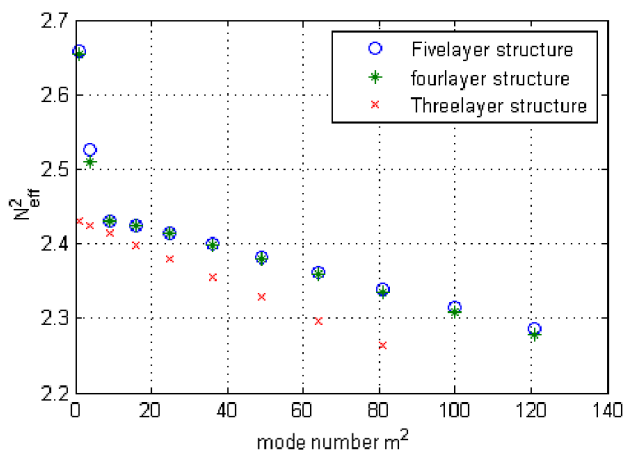

Fig. 6. Theoretical data for structure no. 1.

The data obtained in the experiment for structure no. 1 shows that number of modes increased when second layer of polymer had been inserted. In Fig. 5 we observed that first mode in four-layer structure propagated out of the waveguide layer and two modes in five-layer structure. Theoretical calculation shown in Fig. 6 illustrated that two modes in four- and five-layer structure are propagating out of the waveguide layer.

The structure shown in Fig. 7 contains the same thin layers but the waveguide was made in $\mathrm{Bk} 7$ glass substrate. Theoretical calculations for each layer of the step index waveguide structure are shown in Fig. 8. Table III presents the thickness and the refractive index of each layer.

Structure no. 2 (Fig. 7) has a different substrate (with higher refractive index) and in this case we do not ob-

TABLE II

Thickness and refractive index for structure no. 1.

\begin{tabular}{c|c|c}
\hline \hline & Structure no. 1 (PS+PVDF) \\
& $\begin{array}{c}\text { Thickness } \\
(W \pm \Delta W)[\mu \mathrm{m}]\end{array}$ & $\begin{array}{c}\text { Refractive index } \\
n \pm \Delta n\end{array}$ \\
\hline waveguide & $6.53 \pm 0.20$ & $1.559 \pm 0.030$ \\
layer 1 & $1.175 \pm 0.022$ & $1.645 \pm 0.003$ \\
layer 2 & $0.50 \pm 0.01$ & $1.42 \pm 0.01[7]$
\end{tabular}

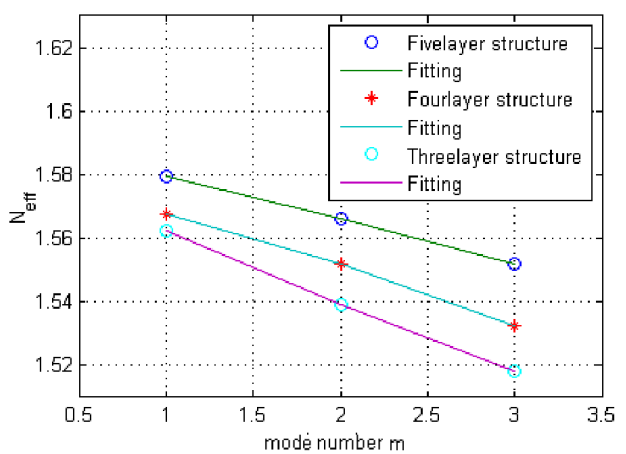

Fig. 7. Experimental data for structure no. 2 made in Bk7 glass substrate.

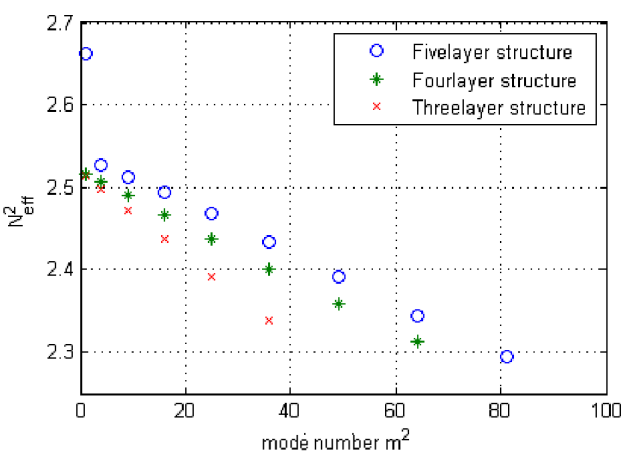

Fig. 8. Theoretical data for structure no. 2.

serve changes in number of modes. We observe propagation outside of first mode of four-layer structure and two modes of five-layer one. Figure 8 (theoretical calculations for the structure of the step-index waveguide) shows two modes propagating outside only in five-layer structure. However, the theoretical calculations (Fig. 8) show that the number of modes increases with the deposition of successive layers.

In the next structure we used different polymer, polyimide (with much higher refractive index) for second layer. The first layer, as before, is made from PVAC.

Calculated effective indices of propagation for structure no. 3 are shown in Fig. 9. Substrate in this structure was made of Gevert's glass. The values of parameters for structure no. 3 and its theoretical $N_{\text {eff }}$ are illustrated accordingly in Table IV and Fig. 10.

TABLE III

Thickness and refractive index for structure no. 2 .

\begin{tabular}{c|c|c}
\hline \hline & Structure no. 2 (PS+PVDF) \\
\hline & $\begin{array}{c}\text { Thickness } \\
(W \pm \Delta W)[\mu \mathrm{m}]\end{array}$ & $\begin{array}{c}\text { Refractive index } \\
n \pm \Delta n\end{array}$ \\
\hline waveguide & $4.18 \pm 0.10$ & $1.587 \pm 0.015$ \\
layer 1 & $1.052 \pm 0.012$ & $1.648 \pm 0.002$ \\
layer 2 & $0.50 \pm 0.01$ & $1.42 \pm 0.01[7]$
\end{tabular}




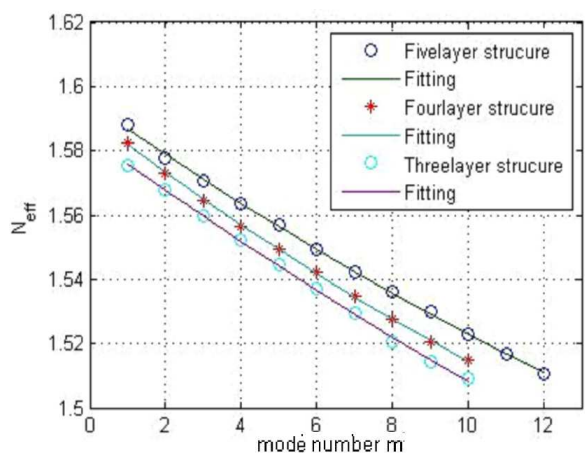

Fig. 9. Experimental data for structure no. 3 made in Gevert's glass.

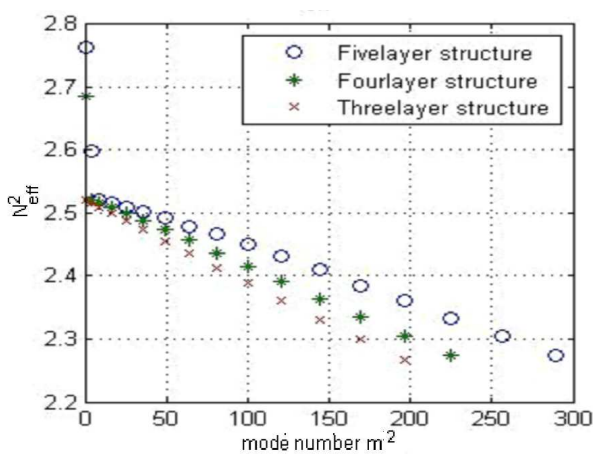

Fig. 10. Theoretical data for structure no. 3.

The gradient index waveguide structure and step index waveguide structure behave similarly. The deposition of the first layer results in one mode propagating out of the waveguide layer but two layers on the waveguide cause propagation of two modes outside.

For the structure with the same polymers configuration but on Bk7 glass as a substrate, the experimental values of $N_{\text {eff }}$ are shown in Fig. 11. The calculated thicknesses and refractive indices of the layers are illustrated in Table $\mathrm{V}$ and theoretical data of step index waveguide structure in Fig. 12.

The deposition of the first layer on the gradient index waveguide structure on $\mathrm{Bk} 7$ as a substrate results in the propagation of one mode outside the waveguide layer. After depositing on it the next layer, two modes propagate out of this layer. The calculations of the pa-

TABLE IV

Thickness and refractive index for structure no. 3 .

\begin{tabular}{c|c|c}
\hline \hline \multicolumn{3}{c}{ Structure no. 3 (PVAC+polyimide) } \\
\hline & $\begin{array}{c}\text { Thickness } \\
(W \pm \Delta W)[\mu \mathrm{m}]\end{array}$ & $\begin{array}{c}\text { Refractive index } \\
n \pm \Delta n\end{array}$ \\
\hline waveguide & $8.45 \pm 0.20$ & $1.588 \pm 0.016$ \\
layer 1 & $1.024 \pm 0.012$ & $1.634 \pm 0.002$ \\
layer 2 & $0.50 \pm 0.03$ & $1.700 \pm 0.001[7]$
\end{tabular}

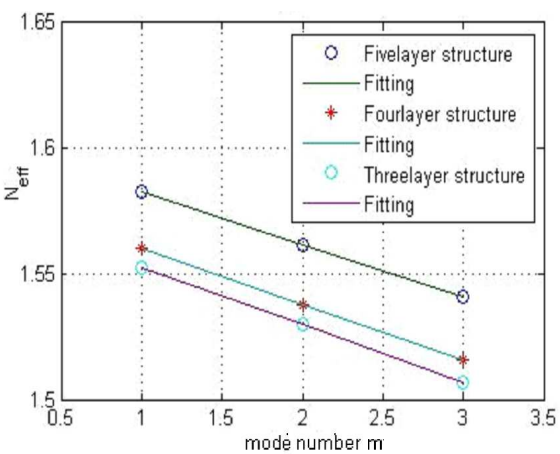

Fig. 11. Experimental data for structure no. 4 made from Bk7 glass.

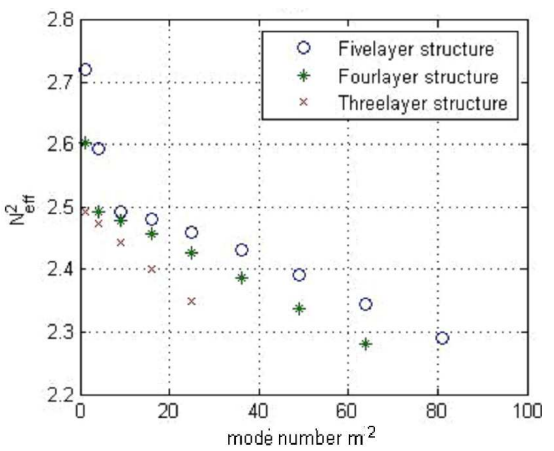

Fig. 12. Theoretical data for structure no. 4 .

rameters of the step index waveguide structure show the propagation outside of two modes after depositing of the first layer and three after depositing on it of the second layer.

The last measured structure had polymers configuration consisting of PS and polyimide.

Data from this structure is shown in Fig. 13. Gevert's glass was a substrate for above structure.

Theoretical values for each layer of the step index waveguide structure are shown in Fig. 14. Table VI contains calculated thickness and refractive index of each layer.

For the gradient index waveguide structure the deposition on the waveguide of the first and on it the second layer causes the propagation outside of only one mode. However, for the step index waveguide structure it results in an increase to two modes propagating out of the

TABLE V

Thickness and refractive index for structure no. 4 .

\begin{tabular}{c|c|c}
\hline \hline \multicolumn{3}{c}{ Structure no. 4 (PVAC+polyimide) } \\
\hline & $\begin{array}{c}\text { Thickness } \\
(W \pm \Delta W)[\mu \mathrm{m}]\end{array}$ & $\begin{array}{c}\text { Refractive index } \\
n \pm \Delta n\end{array}$ \\
\hline waveguide & $3.78 \pm 0.10$ & $1.581 \pm 0.015$ \\
layer 1 & $1.001 \pm 0.01$ & $1.632 \pm 0.002$ \\
layer 2 & $0.50 \pm 0.03$ & $1.700 \pm 0.001[7]$
\end{tabular}




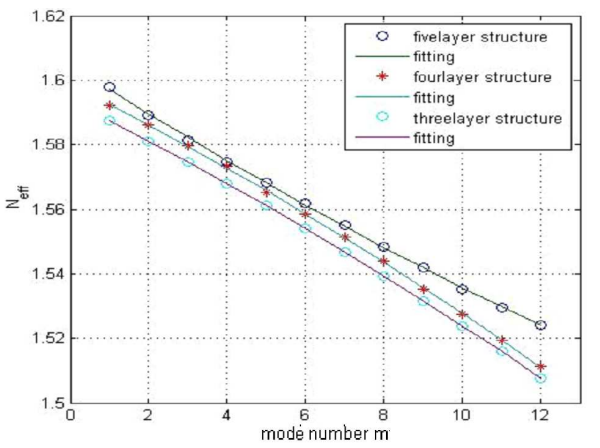

Fig. 13. Experimental data for structure no. 5 made from Gevert's glass.

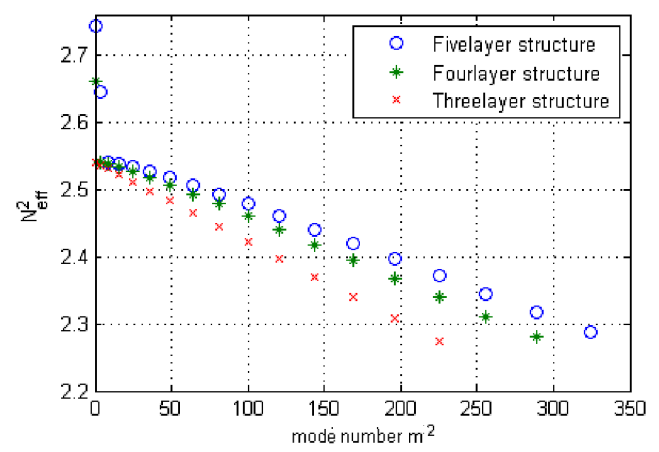

Fig. 14. Theoretical data for structure no. 5 .

waveguide layer.

\section{Conclusion}

In the paper the gradient waveguide with one and two thin layers deposited on it has been studied. Refractive indices of the substances of layers are different: smaller or greater than the one of the waveguide. In all cases the deposition of the thin layer on the waveguide increases the values of effective propagation index (of $m$-mode) irrespective of the difference of the waveguide with the layer $[10,11]$. It was caused by different positions of atoms in thin layers than in bulk substance, so thin layers have different properties. However, in our experiments the difference between PS refractive index and PVAC refractive index was kept, but both layers had a refractive index larger than the waveguide refractive index.

TABLE VI

Thickness and refractive index for structure no. 5 .

\begin{tabular}{c|c|c}
\hline \hline & Shickness \\
& $(W \pm \Delta W)[\mu \mathrm{m}]$ & $\begin{array}{c}\text { Refractive index } \\
n \pm \Delta n\end{array}$ \\
\hline waveguide & $8.89 \pm 0.20$ & $1.594 \pm 0.030$ \\
layer 1 & $1.054 \pm 0.055$ & $1.638 \pm 0.004$ \\
layer 2 & $0.50 \pm 0.03$ & $1.700 \pm 0.001[7]$
\end{tabular}

With the refractive index of the first layer not much higher in relation to the waveguide and the lower refractive index layer on the other layer (structure no. 1 and 2), the second one does not stop the flow of light outside the waveguide.

However, with the refractive index of the first layer not much higher in relation to the waveguide and the much higher refractive index of second layer for increased coefficient of substrate $(\mathrm{Bk} 7)$ there are cases of propagating out of the light from the waveguide.

It can be concluded that the multilayer structure made on the gradient index waveguide is better than the structure made on the step index waveguide because of a smaller number of modes propagating out of the waveguide into the adjacent layer.

The accuracy is not so good for the upper layer; however, the error remains smaller than $1 \times 10^{-3}$ for the refractive index and below $0.2 \mu \mathrm{m}$ for the thickness when uncertainty on the measured coupling angles remains smaller than $0.01^{\circ}$.

The changes in propagation of a light beam not only in waveguide (several modes) layer may be applied to sensing and controlling the direction of light in the waveguide structure (by depositing on it a polymer layer with the appropriate refractive index).

\section{References}

[1] R. Urlich, R. Torge, Appl. Opt. 12, 2901 (1973).

[2] A. Kieżun, T. Patej, H. Działak, Bull. MUT 3, 91 (1981).

[3] N. Uchida, Appl. Opt. 15, 179 (1976).

[4] E. Auguściuk, M. Roszko, Opt. Appl. 31, 377 (2001).

[5] E. Auguściuk, F. Sala, Proc. SPIE 6585, 65852D (2007).

[6] T. Schneider, D. Leduc, C. Lupi, J. Cardin, H. Gundel, C. Boisrobert, J. Appl. Phys. 103, 063110 (2008).

[7] T. Pustelny, M. Grabka, Acta Phys. Pol. A 116, 385 (2009).

[8] http://www.texloc.com/closet/ cl_refractiveindex.html.

[9] M. Blahut, D. Kasprzak, Acta Phys. Pol. A 116, 257 (2009).

[10] D. Dziąg, M.Sc. Thesis, Warsaw University of Technology, Warsaw 2010.

[11] E. Auguściuk, G. Biniecki, Phot. Lett. Poland 1, 124 (2009). 